\title{
Cross Layer Congestion Control in MANETs and Current State of Art
}

\author{
Prof.K.Srinivas \\ Dept of Computer Science Kottam College of \\ Engineering, Kurnool, India
}

\author{
Prof.A.A.Chari \\ Director (Research studies) \\ Rayalaseema University, Kurnool, India
}

\begin{abstract}
Mobile Ad hoc networks have emerged recently as an important trend of future wireless systems. The evolving wireless networks are seriously challenging the traditional OSI layered design. In order to provide high capacity wireless access and support new multimedia network, the various OSI layers and network functions should be considered together while designing the network. In this paper, we conduct brief discussion on current state of the art and performance optimization challenges like energy efficiency, cross-layer design and its congestion control.
\end{abstract}

\section{INTRODUCTION}

The field of communication has witnessed gigantic advancements in the present times. The buzzword of today is communication with mobility. When it comes to high communication mobility, perhaps the most dominant, flexible and resourceful method is Mobile Ad-Hoc Networks (MANETS). Through this paper, I would like to present the standards and guidelines which are utilized for MANET implementation in tandem with the routing protocols. In addition to this, I would also be focusing on two more parameters which are been widely researched.-

1) The cost metrics that define the efficiency and performance of an ad-hoc network

2) The methods to decrease power consumption in of MANETs of ad-hoc networks in a manner that will increase the longevity and maintenance of the mobile ad hoc networks.

But before that we need to know the essential characteristics of a mobile ad hoc network (MANET). MANETs are independent systems of mobile nodes which form a wireless network of sorts where mobile nodes dynamically structure a network to exchange information without making use of any pre-existing fixed network infrastructure.

A MANET is said to exist when a willing node sends data to another willing node who readily accepts it. These mobile nodes play a two-fold role by acting both as hosts and as routers. This helps in the forwarding of packets for other mobile nodes in the network that are beyond the transmission range of the mobile source node. All the nodes of the network play an active role to permits the ad-hoc routing protocol to locate multi-hop paths across the network to any other node.

MANETs are self-configuring, standalone node and router networks that move about freely, organize themselves randomly and are interconnected by wireless links that when synchronized, form a dynamic topology. These make them an ideal for several kinds of applications such as WLANs and
WLLs etc. because they have the ability to establish temporary communication sans any pre-installed infrastructure.

The transmission range of a mobile node in the network is restricted to the node's circular dimensions, the radius of which is based on factors such as the power that is transmitted, sensitivity of the receiver and propagation loss model. The mobile ad hoc network will behave like a multi hop network with multiple nodes acting as routing nodes if the destination node is not confined within the transmission range of the source node. Because of the restricted wireless transmission ranges of each node, data packets are forwarded along multi-hop paths. The three kinds of traffic in MANETS are:

i) Peer to Peer: Communication between two nodes with one hop.

ii) Remote to Remote: Communication beyond one hop but existence of stable route.

iii) Dynamic Traffic: Nodes are dynamic and routes are reconstructed frequently.

\section{OVERVIEW OF CONGESTION PROBLEM IN WIRELESS AD HOC NETWORK}

This section gives a brief introductory overview of the congestion problem. After a concise sketch of the overall problem, we will give an summary of how congestion is dealt with on the Internet,

the TCP congestion control mechanism, to be exact. Next, we show why mobile ad-hoc networks pose new challenges to congestion control [6].

\subsection{The congestion problem}

Adjustment of the data rate used by each sender in order not to burden the network in a network with shared resources is a prerequisite, where multiple senders contend for link bandwidth. Packets that cannot be forwarded are dropped when they arrive at a router. Accordingly, a surplus quantity of packets heading towards network bottleneck leads to many packet drops. These dropped packets might already have traversed a lengthy network path and thus devoured considerable resources. Besides, retransmission is triggered even more by lost packets which is an indication of additional packets been sent over the network. All these factors cause severe deterioration of the network throughput. The end result is zero data delivery with congestion collapse of the network if no appropriate congestion measures are not take up to control it. Such a situation occurred on the early Internet, leading to the development of the TCP congestion control mechanism [7]. 


\subsection{TCP congestion control}

Congestion control is in the task assigned to the transport layer, in particular, of the Transmission Control Protocol (TCP), on the Internet. TCP merges congestion control with reliability mechanisms which lets congestion control to function without any explicit feedback requirements about the congestion state of the network as well as without direct intermediate node participation. All the TCP does it to monitor occurring packet losses when it wants to detect network congestion.

TCP uses cumulative acknowledgments: when a new segment arrives, a TCP receiver always acknowledges the finish of the correct and complete data received so-far. If out-of-order segments are received, i.e., some data is missing between the acknowledged and the newly arriving data, the last acknowledgment is resent (duplicate ACK).

A window-based additive increase - multiplicative decrease mechanism is employed in a TCP. When no packet losses take place, the window dimension is additively increased by one segment in every round-trip time. Where a duplicate acknowledgment is received, a TCP sender will presume that some packet reordering has taken place in the network. However, a congestion loss is assumed when the fourth copy of an acknowledgment (Triple Duplicate ACK, TDACK) is received. In such a scenario, the missing segment is replicated with the window size cut into half (multiplicative decrease). TCP also uses a timeout that is dependent on the round-trip computed time of the connection. The TCP concludes severe congestion when this retransmission timeout (RTO) elapses sans any acknowledgement. As a result of this, the window size is decreased to one and the unrecognizable segment is resent back. The timeout is doubled until the next retransmission attempt if no acknowledgment is received till then. Thus, there is exponential growth of this timeout.

A mechanism called slow start is engaged, which allows for a speedier convergence to the correct window size, during the first phase of a connection and after a timeout. While slow start is active, the window size is not increased for all the received acknowledgments and not just for one segment size for every round-trip time. This indicates that the size of the window grows exponentially during this phase. Two of the extensions and modifications of TCP are predominantly applicable out of the many proposed because they have been implemented for some MANET congestion control methods. When the delayed acknowledgments (DACK) [8] option is used, an acknowledgment is sent for every second segment only and not for every single segment, or after the expiration of some timeout at the receiver. This leads to the deduction of acknowledgement packets. The selective acknowledgment (SACK) [9] option allows for a more fine-grained feedback on missing segments than the standard cumulative ACK scheme. It is especially supportive if multiple segments in the same round-trip period are amiss.

\subsection{Congestion in mobile ad-hoc networks}

While TCP congestion control is highly efficient over the Internet, MANETs display some exceptional properties that generally affect the design of the appropriate protocols and protocol stacks in a substantial manner and of a congestion control mechanism in particular. The huge environmental disparities in a mobile ad hoc network pose huge problems for standard TCP.

The node mobility and a shared, wireless multi-hop channel are the principal properties of MANETs. Changes in routes are indicative of node mobility and of the intrinsically unpredictable medium which results in unsteady packet delivery delays and packet losses which are not to be construes as congestion losses.

Using a wireless multi-hop channel permits a single data transmission only within the interference range of one node. Hence, geographical close links are dependent on one another thereby influencing the manner in which the network congestion largely manifests itself. A typical Internet router is a dedicated host that is connected by high bandwidth links. Whenever there is Internet congestion taking place, it is generally focused on one single router. On the contrary, MANET congestions affect the entire area due to a shared medium where regions of network and not nodes are congested.

Packet losses, which normally depend on the network type, that are not due to network congestions can be found to occur more frequently in wireless networks. These results in negative reactions of TCP congestion control. The observation of packet losses is very difficult as the transmission times (as well as the round trip times) exhibit a high variation.

A single sender is accidentally or intentionally capable of causing a network collapse due to congestion owing to the relatively lower bandwidth of mobile ad-hoc networks. Severe imbalances can take place between flows due to the severe effect of a single traffic flow on the network condition. Traditional wire line networks like the Internet are not so prone to congestion-related problems as compared to wireless multi hop networks. We, therefore conclude that a balanced congestion control is the foundation for network stability and superior performance.

Because of the heterogenic nature of application scenarios for multi hop wireless networks, suitable congestion control solutions for a specific network and application will mostly depend on the properties and the function of the relevant network. Hence, there would be customized solutions for different scenarios instead of a single, general purpose one as reflected in this paper. A majority of these proposals do not represent complete, ready-to-use protocols, but rather solutions for a subset of the identified problems. These can serve as the basis for application-tailored protocol stacks. A few of the protocol properties are, however, important for a broader range of applications.

\section{CONGESTION CONTROL IN WIRELESS AD HOC NETWORK}

The past couple of years have seen the reception of extensive focus on the problem of congestion control both in the Internet context, in addition to an ad-hoc network context. Much of the research focus has been on modeling, analysis, algorithm development of end-to-end control schemes (such as TCP), and adaptation of such schemes to ad-hoc networks. Algorithms that unite and stabilize operations have been developed, given the routing path and the bandwidth constrains. However, in the context of a wireless network, another main constrain is due to the MAC (Media Access Control) layer. Most wireless MACs utilize a time-division strategy for accessing channel where at 
any point in space, the physical channel can be accessed by a single user at each moment of time (a time constraint). Here, we discuss a few of the existing congestion control techniques in brief:

\subsection{Hop-by-Hop congestion control:}

Extensive research of hop-by-hop congestion control algorithms have been done in the context of the Internet. These schemes give a feedback of the congestion state at a node to the hop preceding it. The preceding node then adjusts its rate of transmission on the basis of this feedback. Based upon the queue length at the congested node, a feedback is provided. Congestion is indicated if the queue length exceeds a threshold. The preceding node is alerted so that transmission rate is reduced. Such schemes, by quickly reacting to congestion rather than to end-to-end schemes (the bottleneck node would send feedback backward, thus decreasing the delay in the control loop), yield more productive output as compared to a corresponding end-toend scheme. Research in alternate mechanisms in the recent past has concentrated more on Internet congestion controlled mainly by end-to-end schemes (in particular, TCP) primarily due to its deployability and scalability. Generation of scalability problems occurs when hop-by-hop schemes are required to have per-flow intermediate node state management. Conversely, the number of flows per node is of a much smaller order than in the Internet when it comes to wireless networks. Wireless networks generally have per-flow queuing as a result of packet scheduling. Because different users are at different locations, different physical layer strategies (such as the channel coding and modulation scheme of the power level) are required. Thus, we argue that hop-by-hop schemes are feasible over a wireless network [10].

\section{ADAPTIVE ROUTING TECHNIQUE FOR CONGESTION CONTROL}

CONGESTION ADAPTIVE ROUTING (CRP): In this control technique, each node that appears on a route will alert the previous node whenever congestion is likely to happen. Once this happens, the previous node uses a "bypass" route to take a detour in order to evade the potential congestion to the first noncongested node on the route. The process will lead to a probabilistic traffic split over the primary and the bypass routes thereby reducing the chances of congestion in an efficient way. $\mathrm{CRP}$ is on-demand and consists of the following components [11]:

\subsection{Congestion Monitoring}

In order for a node to keep a check on the congestion status, a range of metrics can be used. Some of the main ones are the percentage of all packets disposed of due to lack of buffer space, the average queue length; the number of packets timed out and retransmitted the average packet delay, and the standard deviation of packet delay. Whenever the numbers escalate, it indicates that congestion is sprouting up. Either one of these methods can practically work with CRP. The congestions status of nodes can be additionally classified into three levels: "green", "yellow", and "red". A node is said to be "green" if it is far from congested, "yellow" if likely congested, or "red" if most likely or already congested. As discussed at a later stage, a bypass is a path from a node to its next green node. The next green node is the first green node that is at least two hops away downstream on the primary route.

\subsection{Primary Route Discovery}

The sender broadcasts a REQ packet to the receiver to locate a route to the receiver. As a response to the first copy of REQ, an REP packet is then sent to the sender by the receiver. The REP will travel back the same path that was previously taken by the REQ. This path becomes the primary route between the sender and the receiver. Nodes along this route are termed as primary nodes. Two tactics are often employed by us to decrease route discovery traffic and to get a better grip on network congestion problems: (1) the REQ is dropped if arriving at a node already having a route to the destination, and (2) the REQ is dropped if arriving at a node with a "red" congestion status[11].

\subsection{Bypass Discovery}

An UDT (update) packet is regularly broadcast by a node to its neighbors. This packet comprises of the node's congestion status and a set of tuple $f$ destination $R$, next green node $G$, distance to green node $\mathrm{mg}$, each for a destination $\mathrm{R}$ that the node has a route to. The aim of doing this to make node $\mathrm{N}$ become aware of the congestion status of Nnext when $\mathrm{N}$ receives a UDT packet from its next primary node as well as to learn that the next green node is $\mathrm{G}$ which is $\mathrm{m}$ hops away on the primary route. Congestion is likely ahead if data packets continue to be forwarded on link $\mathrm{N}$ $\rightarrow$ Nnext if Nnext is yellow or red. $\mathrm{N}$ starts to discover a bypass route toward node $\mathrm{G}$ - the next green node of $\mathrm{N}$ known from the UDT packet because CRP tries to keep congestion from occurring in the first place. $\mathrm{N}$ begins to ascertain a bypass route toward node $\mathrm{G}$ - the next green node of $\mathrm{N}$ known from the UDT packet. This bypass search is comparable to the primary route search, except that: (1) the bypass request packet's TTL is set to $2 \mathrm{X} \mathrm{m}$, and (2) the bypass request is dropped if arriving at a node (neither $\mathrm{N}$ nor $\mathrm{G}$ ) already present on the primary route. As a consequence, it is more economical to locate a bypass and the bypass be disjointed with the primary route, barring that they join at the end nodes $\mathrm{N}$ and $\mathrm{G}$. There is a probability that no bypass is discovered due to the manner in which the bypass request approaches $\mathrm{G}$ in which case; we go on using the primary route. But we also locate that the chance for a "short-cut" to subsist from one node to another on a route is important.

\subsection{Traffic Splitting and Congestion Adaptability}

The probability $\mathrm{p}$ to forward data on the primary link is set to 1 at the beginning (i.e., no data is sent along the bypass) at each node that has a bypass. Periodical modification is done on the basis of the congestion status of the next primary node and the bypass route. The congestion status of the bypass is the accumulative status of every bypass nodes. The key is that we should increase the amount of traffic on the primary link if the primary link leads to a less congested node and reduce otherwise. An example is demonstrated by Figure 1, where the bypass from $\mathrm{A}$ is $\mathrm{A} \rightarrow \mathrm{X} \rightarrow \mathrm{Y} \rightarrow \mathrm{C}$, from $\mathrm{B}$ is $\mathrm{B} \rightarrow \mathrm{Y} \rightarrow \mathrm{Z} \rightarrow \mathrm{E}$, and from $\mathrm{D}$ is $\mathrm{D} \rightarrow \mathrm{W} \rightarrow \mathrm{F}$.

\subsection{Multi-path Minimization}

CRP endeavors to reduce the use of multiple paths so as to minimize the protocol overheads. If the probability $\mathrm{p}$ to forward data on a primary link nears the value of 1.0 , it indicates that the next primary node is far from congested or the bypass route is extremely congested. In such a scenario, the current node bypass is eliminated. Likewise, if the next primary node is highly congested ( $\mathrm{p}$ approaches 0 ), the primary link is disengaged and the bypass route becomes primary. CRP permits a node to have 
only one bypass in order to make the protocol very lightweight. By making use of a bypass, the protocol overheads are partially minimized due to short bypass lengths. Each bypass connects to the first non-congested node after the congestion spot, which should be just a few hops downstream.

\subsection{Failure Recovery}

CRP is a popular routing protocol that is capable of elegantly and swiftly recommencing connectivity after a link breakage by taking the help of currently available bypass routes.

\section{CURRENT STATE OF THE ART}

Akyol, $\mathbf{U}$ et al.,[20] studied a jointly performing scheduling and congestion control in mobile ad-hoc networks. Network queues remain bounded and the resulting flow rates satisfy an associated network utility maximization problem was the conclusion of the proposal. They defined a specific network utility maximization problem and argued is appropriate for mobile ad hoc networks. They described a wireless greedy primal dual (wGPD) algorithm for combined congestion control and scheduling in wireless ad-hoc networks and compared its performance with the standard 802.11+TCP protocols.

Observation: The spectrum is limited in most of the ad hoc networks, so that these ad hoc networks would benefit most from the performance gains of wGDP. But the proposed wGDP limited to unicast models. This wSDP need to be extend and verified for multicast traffics. wGDP also need to evaluate in the context of power and rate control

Ahmed S. Nagy et al., [15] argued that due to mobility of nodes, and less power processing capability, manet is facing challenges those causes reduction in the lifetime of a node which increases cost parameters and decreases reliability parameters. The authors attempted to derive as solution to increase the lifetime of Ad hoc networks. They tried to achieve this by using Multi-path congestion and Power aware routing protocols".

To achieve the aim, developed protocols are SWARM-BASED routing and helped in incorporating power awareness and load balancing techniques. Here in the paper, swarm based techniques are efficiently defined as that is to define algorithms, systems or distributed problem solving architecture inspired from animal colonies like ants, bees etc. This paper is developed based on ANT-BASED swarm technique for implementing POWERANT process. SWARM intelligence techniques of ant are used here to achieve the goal of load balancing and power consumption. The novelty of the proposal is all 2 ideas that

1) Identifying the dynamic nodes which are is our area of interest.

2) The challenges the network is facing due to this less power capacity and lack of load balancing and to try solving it through swarm techniques instead of increasing the number of nodes.

In fact, the working procedure of the ant-based swarm technique is very interesting:

a. In basic, 1st the node initiated a packet called ANT and sends to forward nodes with an idea of gathering information about those nodes. b. The ant, in the process of journey, takes all power metric values of nodes and stores for future calculation of better route for packets.

c. The pheromone table which initially stores the values of power metric at each node are updated by ant's results.

d. The sent ant returns the same node with information to update and this retrieving ant is called back ant.

e. The forwarded ant reaches all the nodes in its transmission range, and all remaining nodes are termed switch off.

This entire study is very interesting and good. The proposal also discussed well and gave a clear idea on

- Route-Discovery Process,

- Structure of discovered packet,

- How to process a forward ant,

- $\quad$ Structure of Pheromone table entry

- Processing a back word ant in all conditions.

The author also made design discussions which are productive and serial. In addition, presentation of system architecture and Routing algorithm is done efficiently. Eventually, the paper is able to solve the problem to low power capacity of nodes in AD hoc networks. The developed system is able to solve the MANET routing protocol issues. The main achievements of the work is

- Incorporating power metric in routing

- Enforcing load balancing

- Making best use of load balancing.

As entire result, lifetime increased by $33 \%$, decreased power skewness and cost parameters per byte.

Observation: The proposed model not considered an issue that incorporating incentive which helps the node in future to give value, basing on social helping. Another important factor it also has to focus on is controlling the QUALITY OF SERVICE. On a whole the work of paper is good and should concentrate on the above left areas.

The chances of success probability for transmission over wireless link are dependent on power given and scheduled transmission rate. But the problem to overcome is to get decentralization which is possible for the case of parallel channels. Algorithmic suggestions can be found for zero-full power allocations and by solving a maximum weighted matching problem over a conflict graph. Anastasios Giovanidis et al.,[16] attempted to achieve fully distributed implementation by approaching the second problem with the arsenal of super modular game theory, where time (t), destination nodes (d) and also predefined destination denoted by (s) will be divided. After solving and finalizing the model crawl up to two theorems those follows.

Theorem 1: The capacity region A of the wireless network under study is the set of all non-negative vectors $\mathrm{x}==(\mathrm{Xl}, \ldots, \mathrm{Xs})$ (each element of which denotes an incoming average data rate entering at node s E S and having destination $d$ such that there exist multi commodity good put flow variables satisfying being, the optimal policy having achieved stability for vectors with 
name "GOODPUT BACKPRESSURE POLICY" and this good put region equals convex hull and then followed by the properties of success function

1) $\mathrm{ql}$ is strictly increasing in $\mathrm{Pl}$ and the $\log$ of the function is concave in $\mathrm{Pl}$

2) ql is strictly decreasing and convex in $\mathrm{Pk}, \mathrm{Vk}$ il, $\mathrm{kE} \mathrm{L}$

3) The log of the function has increasing differences for each pair of variables.

Theorem 2 the success probability function for the Rayleigh / Rayleigh fading case, given satisfies the above properties.

Proof: For the proof, the expressions of first and second order partial derivatives are required. That too, from the function is increasing in $\mathrm{Pl}$ and decreasing in $\mathrm{Pj}$ i.e., the achievement of Rayleigh's fading case that satisfies above properties and then its properties are seen followed by NUM problem based on dual composition in which there are inputs rate controlled problems solved for each commodity flow at the incoming nodes independently Xs == Us' -1 (As). Observe that by assumption $\mathrm{Us}^{\prime}(\mathrm{xs})$ is continuous and monotone decreasing in IR+ (thus bisection) and the inverse of the function exists.

Since Us (xs) is strictly concave the solution is unique for each As and the scheduling problems The solution of (15) further provides the optimal multi commodity good put.

The scheduling problem consists of several other factors like relaxation, which further deviates to two more assumptions, they are:- Assumption 1: Each origin node chooses a single end node to transmit Assumption 2: Each node can transmit and receive at the same time, optimality conditions which explains about the divisions, a super modular game in which the discussions is about "POWER PLAYER" who belong to the set NP, each one of which represents a node and the set of feasible joint strategies IIP is identical to the set II of feasible power allocations. and "PRICE PLAYER" in which we prove them with theorems, this is then tailed with the scheduling algorithm having two phases, the best being "POWER UPDATE" phase, followed by implementation issues which allows scheduling algorithm to have well based applications where topology may change due to user to user modifications or as per requirement which is finally followed by stimulations which result for congestion control, routing, distributed power allocation when hop-by-hop retransmission is considered. A remark to two solutions would be exactly same if objective for would be concave.

Observation: The two conditions, i.e., necessary condition, if the network is stable and sufficient condition i.e., if the system is stable under good put backpressure policy and this proof utilizes the Foster - Lyapunov stability criteria the data arrivals per slot $\mathrm{a} \sim(\mathrm{t}+1)$ are realizations of i.i.d. random and the power allocation is based solely on the information on the current queue lengths and finally the random variables $\mathrm{X}$, are analyzed for and secured.

Fatma Outay_y et al., [17] proposed a model towards an efficient Energy-Aware Service And Route Discovery scheme in MANET, the authors, "effect of various methodologies over energy engross of the instruments when IETFs MANET is used" and "testing if the Service Discovery Efficiency is economic for both cross-layer protocols" is dealt. This model also deals with the facility of rendering a cross-layer Service Discovery. This scheme regards to minify the engrossing of energy. It provides an Energy-Aware Service and Route Discovery Scheme called
"EASARD". In this paper, this scheme is proposed depicting its effectiveness and it is compared regarding engrossing of energy with other "Integrated Protocol". The routing discovery scheme includes enhancing "Zone Routing Protocol" and embedding operating data within the directing protocols. The effect of various technologies over the energy engrossment of roving instruments is enhanced in this model. This method is sophisticated over cross-layer schemes which can be seen through the large modeling nature. The key aim of this proposal is to project the "scalability" and "affectivity" of the protocol efficiency assessment. For projecting the performance, the power engrossment and operation invention are considered.

Here, Bloom Filters which are utilized for explaining the operating data embedded in directing messages are explained clearly along with their IARP packet Format. The architecture and organization of the Jist/Swans is depicted in this paper. Jist/Swans simulator is an energy model utilized to compute the total power engrossment. They used this model and its changed version to enhance the integrated protocol. This is done by computing the average of invention engrossed power for every node.

The performance of the proposed methodology is explained using OLSR directing protocol (Optimized Link State Routing Protocol). Then, the advanced version of OLSR, called SDOLSR which is an integrated methodology is explained. These techniques are employed in the directing layer. The EASARD protocol and SD-OLSR are distinguished and analyzed.

Many attributes are modeled to distinguish between EASARD and SD-OLSR which is done by utilizing Jist/Swans model. The parameters undertaken are Simulation area, simulation time, data rate, transmission range, Mac Layer, Wi-Fi radio bandwidth, zone radius, services offered, start up power, transmission power, reception power and the idle power.

The "hybrid directing protocol" called "ZRP" is integrated with the operation invention for every change. This combination is related and analyzed with the conventional directing protocol. The conventional protocols don't have the provision of "piggybacked" operation data. After comparing to the conventional protocol, it is distinguished with the "pro-active integrated protocol" called "SD-OLSR". The considered attributes here are their Energy effectiveness and the Economy. During the enhancement of EASARD, affect of several constraints are considered which include network density, node speed and zone radius. Whereas, while comparing EASARD and SD-OLSR, affect of network density, bit rate and node speed are explained.

While considering the attribute, evaluation of EASARD, the proposed model is related and analyzed with the standard ZRP protocols. It is done so to depict that piggybacking doesn't increment the energy engrossment and graphs are drawn related to them. The graphs drawn depict the similar curves and thus proving that piggybacking has no affect over energy engrossment. In the second consideration, this paper deals with relating and distinguishing EASARD with SD-OLSR within similar surroundings and considering similar parameters. This is done so to demonstrate that the proposed methodology is a better choice.

While considering second attribute the mean of invented operations per node and assessing their affect over the energy engrossment are computed. Here, the operation invention Cost in idle network and dynamic network are considered and 
compared. The parameters such as comparative quantity of broadcast and peer-to-peer network, packet quantity and their dependency over "promiscuous mode operation" are considered during the architecture and organization of the energy alert protocol.

The proposed methodology depicts an organization which combines service discovery operation and the conventional directing protocols. This is used to explain the difficulty of energy engrossment. Also, the conventional methodologies face ambiguity of packet routing at the time of operating and directing operations at the "Application layer". These operations are controlled by protocols used for accessing and finding data. EASARD is used to find the energy performance and affect of network density and node roving over it.

Test results projected by authors, which regularly operate equal integrated operation invention. These rely over "Pro-active directing protocol" and operate in both static and dynamic surroundings. EASARD explained, minify energy engrossment remarkably. It is also used to convey more operation invention rate. In the proposed methodology, operation invention protocol is combined with "hybrid" directing.

Observation: The authors evaluated the proposed EASARD protocol to show its gain in terms of energy consumption and service availability in different conditions, but they limited only to compare it to another similar but pro-active cross-layer protocol. It is necessary to compare with an application layer protocol to demonstrate its superiority in energy efficiency over all other service discovery protocols.

T.Senthil kumaran et al.,[18] presented an early detection congestion and control routing protocol for wireless Ad-hoc networks called as EDAODV which detects congestion at a node level by calculating queue status value and finding congestion status. These Ad hoc mobile networks are made up of fine mobile nodes which communicate through a wireless medium which do not come fortified with any fixed backbone infrastructure. Congestion, which mostly occurs in those intermediate nodes, is prone to heavy data loss. The authors here presented a novel ideology in order to overcome this backdrop.

The process finds more non-congested alternate paths and chooses a best single path. Ad-hoc wireless networks are usually defined as an autonomous system of nodes connected by wireless links communicating in a multi-hop fashion whose main objective is to limit the delay and buffer overflow. Routing protocols of ad-hoc networks can be divided into proactive, reactive and hybrid protocols.

The main motivation of this paper is that congestion is a main cause for packet loss in MANET's. Early detection of congestion and control routing protocol is a unicast routing protocol for MANET. A complete design with an in depth evaluation for this routing protocol is presented in this paper elaborately. EDAODV comprises of the following components:

i. Route discovery which discovers route to the destination and broadcasts an RREQ packet toward the destination, the destination in return broadcasts an RREP back in the same path.

ii. Early Congestion Detection: another component of the EDAODV, which detects the early congestion by means congestion metric at a node. According to the authors a primary path of a node predicts its congestion status and periodically broadcasts a congestion status packet, due to the congestion at the primary route an alternate path was formed at the node. This is attributed under the bidirectional property of the EDAODV.

The congestion mechanism extends further into more of these as stated below:

\section{A. AODV Routing Protocol \\ B. Early Congestion Detection Techniques \\ C. Bidirectional path discovery}

Performance metrics like packet delivery ratio, end to end delay; simulation configuration; varying number of connections; varying CBR load; comes as a part of the performance study which compares EDAODV's performance to AODV routing protocols in MANET. EDAODV is a predictive congestion and control routing protocol in MANETs which had lost fewer packets than AODV that are not having congestion control mechanism because it tries to detect congestion in advance from occurring in the first place, rather than dealing with it reactively.

Observation: The bi-directional alternate paths discover concept of EDAODV design tries to find out non-congested alternate path and avoid congestion in the network. Their ns-2based simulation has confirmed that the advantages of EDAODV demonstrated a significantly improvement of end-toend delay and packet delivery ratio over AODV. Currently they are implementing the EDAODV algorithm in the ns-2 simulator and in future they will be extended to other networks like WMN and WSN and study its performance.

Rahim Rashidi et al.,[19] proposed a new protocol based on congestion control is laid stress on. All factors such as control of workload, stability and transfer on highly secured paths. Each node creates and controls the functioning of the networks termed as ad-hoc networks. Although the topology is dynamic it forms routes. All the nodes that act as the mobile routers control the entire message that pass by them. Some major problems do often occur in this method and hence some malicious nodes do occur. These malicious nodes will be carryout a series of attacks against the routing protocols to facilitate route serving. The model thus presented is categorized into three components: Trust agent, Reputation agent and Combiner agent. The major task of these nodes is to transfer packets only to those secured nodes available in the path. Thus the developed trust protocol is based on congestion control. Despite the method of sequential numbers in control packets it suffers two major attacks:

There is a high probability of malicious nodes to increase its number in this technique. This may lead to fallacious routes producing the least possible count. The model presented in this paper is classified into four components; Trust agent, Reputation agent, combiner agent and congestion control agent. Trust agent controls the level of trust at each node. Reputation node carries forward the message to all other nodes in the network. Combiner agent takes charge of trust received from the above two agents. And as per the information from combiner agent the congestion control agent loads message on all superior trust nodes. The main job of trust agent is to provide: Trust Derivation Trust Quantification and Trust Computation. The reputation agent performs its sharing task based on two popular techniques: Integrated Reputation Exchange and Independent Reputation Exchange. The combiner agent compares the above two agents' 
response and hence total trust value for each node is listed. This is done by a computer based technique on probability.

The congestion control agent considers the node energy for distributing load on the nodes with greater trust values. If the trust value of a node doesn't change within a desired time a node with lesser trust value is chosen. In the thesis two basic methods to set the congestion control agent are discussed. One method discussed is to find the highest amount trust node that does not change within the set time limit. In the AODV protocol proposed in the paper, the table is firstly read to find a route to the destination. In the cases where no routes are found, the ROUTE REQUEST packet is then set to find the respective node. After the search for the respective route is finished the path with least cost is determined. Therefore as per the routing table each node sends or forwards a packet to next node. In cases where routes are not found a ROUTE ERROR packet is put through the network. The model proposed describes the trust derivation method for each node and with the help of Packet
Acknowledgement the necessary data about transmission of packets is determined. Trust Quantification is the one that computes situational trust values for each node is determined. The Trust Computation computes the level for each node based on the categories such as Pp, GR, BL, and HM.

Observation: The model presented introduces the protocols to determine high trust value nodes and hence congestion could be performed. It ensures distribution of the entire work load on the various nodes with higher trust values that are defined by congestion control technique. With the help of low congestion, transfer of data over trusted nodes is possible. From the model presented they have concluded that in spite of some malicious nodes and congestion it is possible to improve the throughput for any network.

\begin{tabular}{|c|c|c|c|c|c|c|}
\hline \multirow{2}{*}{$\begin{array}{l}\text { Protocol that deals with } \\
\text { congestion } \\
\text { Protocol }\end{array}$} & \multicolumn{6}{|c|}{ Application scenario that causes congestion } \\
\hline & $\begin{array}{l}\text { Route } \\
\text { Failures }\end{array}$ & \begin{tabular}{|l|}
$\begin{array}{l}\text { Wireless } \\
\text { loses }\end{array}$ \\
\end{tabular} & $\begin{array}{l}\text { shared } \\
\text { medium }\end{array}$ & $\begin{array}{l}\text { Acknowledg } \\
\text { ement traffic }\end{array}$ & \begin{tabular}{|l|} 
Output \\
Limits \\
\end{tabular} & $\begin{array}{l}\text { Alternative } \\
\text { protocol usage }\end{array}$ \\
\hline TCP-F & YES & $\mathrm{NO}$ & NO & NO & $\mathrm{NO}$ & NO \\
\hline ELFN & YES & $\mathrm{NO}$ & $\mathrm{NO}$ & $\mathrm{NO}$ & $\mathrm{NO}$ & $\mathrm{NO}$ \\
\hline TCP-BuS & YES & $\mathrm{NO}$ & $\mathrm{NO}$ & $\mathrm{NO}$ & $\mathrm{NO}$ & NO \\
\hline Fixed RTO & YES & $\mathrm{NO}$ & $\mathrm{NO}$ & $\mathrm{NO}$ & $\mathrm{NO}$ & $\mathrm{NO}$ \\
\hline ENIC & YES & $\mathrm{NO}$ & $\mathrm{NO}$ & $\mathrm{NO}$ & $\mathrm{NO}$ & $\mathrm{NO}$ \\
\hline TCP-RC & YES & $\mathrm{NO}$ & $\mathrm{NO}$ & $\mathrm{NO}$ & $\mathrm{NO}$ & $\mathrm{NO}$ \\
\hline ATCP & YES & $\mathrm{NO}$ & $\mathrm{NO}$ & $\mathrm{NO}$ & $\mathrm{NO}$ & $\mathrm{NO}$ \\
\hline Cross-layer information awareness & YES & $\mathrm{NO}$ & $\mathrm{NO}$ & NO & $\mathrm{NO}$ & NO \\
\hline TCP-DOOR & YES & $\mathrm{NO}$ & $\mathrm{NO}$ & $\mathrm{NO}$ & $\mathrm{NO}$ & $\mathrm{NO}$ \\
\hline Preemptive routing & YES & $\mathrm{NO}$ & $\mathrm{NO}$ & $\mathrm{NO}$ & $\mathrm{NO}$ & $\mathrm{NO}$ \\
\hline Atra & YES & $\mathrm{NO}$ & $\mathrm{NO}$ & $\mathrm{NO}$ & $\mathrm{NO}$ & $\mathrm{NO}$ \\
\hline Signal-strength based link management & YES & $\mathrm{NO}$ & $\mathrm{NO}$ & $\mathrm{NO}$ & $\mathrm{NO}$ & $\mathrm{NO}$ \\
\hline Multipath-TCP & YES & $\mathrm{NO}$ & $\mathrm{NO}$ & $\mathrm{NO}$ & $\mathrm{NO}$ & $\mathrm{NO}$ \\
\hline TCP/RCWE & $\mathrm{NO}$ & YES & $\mathrm{NO}$ & $\mathrm{NO}$ & $\mathrm{NO}$ & $\mathrm{NO}$ \\
\hline ADTCP & $\mathrm{NO}$ & YES & $\mathrm{NO}$ & $\mathrm{NO}$ & $\mathrm{NO}$ & $\mathrm{NO}$ \\
\hline ADTFRC & $\mathrm{NO}$ & YES & $\mathrm{NO}$ & $\mathrm{NO}$ & $\mathrm{NO}$ & $\mathrm{NO}$ \\
\hline Edge-based approach & YES & NO & NO & NO & $\mathrm{NO}$ & $\mathrm{NO}$ \\
\hline LRED / adaptive pacing & $\mathrm{NO}$ & $\mathrm{NO}$ & YES & $\mathrm{NO}$ & $\mathrm{NO}$ & $\mathrm{NO}$ \\
\hline NRED & $\mathrm{NO}$ & $\mathrm{NO}$ & YES & $\mathrm{NO}$ & $\mathrm{NO}$ & $\mathrm{NO}$ \\
\hline COPAS & $\mathrm{NO}$ & $\mathrm{NO}$ & YES & $\mathrm{NO}$ & $\mathrm{NO}$ & $\mathrm{NO}$ \\
\hline \multicolumn{7}{|l|}{ CAR } \\
\hline Split-TCP & $\mathrm{NO}$ & $\mathrm{NO}$ & YES & $\mathrm{NO}$ & $\mathrm{NO}$ & $\mathrm{NO}$ \\
\hline Alleviating self-contention & $\mathrm{NO}$ & $\mathrm{NO}$ & YES & $\mathrm{NO}$ & $\mathrm{NO}$ & $\mathrm{NO}$ \\
\hline OPET & $\mathrm{NO}$ & $\mathrm{NO}$ & YES & $\mathrm{NO}$ & $\mathrm{NO}$ & $\mathrm{NO}$ \\
\hline RE-TFRC & $\mathrm{NO}$ & $\mathrm{NO}$ & YES & $\mathrm{NO}$ & $\mathrm{NO}$ & $\mathrm{NO}$ \\
\hline Dynamic delayed ACK & $\mathrm{NO}$ & $\mathrm{NO}$ & $\mathrm{NO}$ & YES & $\mathrm{NO}$ & $\mathrm{NO}$ \\
\hline Dynamic adaptive ACK & $\mathrm{NO}$ & $\mathrm{NO}$ & $\mathrm{NO}$ & YES & $\mathrm{NO}$ & $\mathrm{NO}$ \\
\hline Preferred ACK Retransmission & $\mathrm{NO}$ & $\mathrm{NO}$ & $\mathrm{NO}$ & YES & $\mathrm{NO}$ & $\mathrm{NO}$ \\
\hline Combining data and ACK packets & $\mathrm{NO}$ & $\mathrm{NO}$ & $\mathrm{NO}$ & YES & $\mathrm{NO}$ & $\mathrm{NO}$ \\
\hline Dynamic congestion window limit & $\mathrm{NO}$ & $\mathrm{NO}$ & $\mathrm{NO}$ & $\mathrm{NO}$ & YES & $\mathrm{NO}$ \\
\hline SCA & $\mathrm{NO}$ & $\mathrm{NO}$ & $\mathrm{NO}$ & $\mathrm{NO}$ & YES & $\mathrm{NO}$ \\
\hline FeW & $\mathrm{NO}$ & $\mathrm{NO}$ & $\mathrm{NO}$ & $\mathrm{NO}$ & YES & $\mathrm{NO}$ \\
\hline Non-work-conserving scheduling & $\mathrm{NO}$ & $\mathrm{NO}$ & $\mathrm{NO}$ & $\mathrm{NO}$ & YES & $\mathrm{NO}$ \\
\hline RBCC & $\mathrm{NO}$ & $\mathrm{NO}$ & $\mathrm{NO}$ & $\mathrm{NO}$ & YES & $\mathrm{NO}$ \\
\hline С3ТCP & NO & $\mathrm{NO}$ & $\mathrm{NO}$ & $\mathrm{NO}$ & YES & $\mathrm{NO}$ \\
\hline TCP-AP & $\mathrm{NO}$ & $\mathrm{NO}$ & $\mathrm{NO}$ & $\mathrm{NO}$ & YES & $\mathrm{NO}$ \\
\hline EXACT & $\mathrm{NO}$ & $\mathrm{NO}$ & $\mathrm{NO}$ & $\mathrm{NO}$ & $\mathrm{NO}$ & YES \\
\hline
\end{tabular}




\begin{tabular}{|l|l|l|l|l|l|l|}
\hline ATP (Sundaresan) & NO & NO & NO & NO & NO & YES \\
\hline ATP $($ Liu $)$ & NO & NO & NO & NO & NO & YES \\
\hline WXCP & NO & NO & NO & NO & NO & YES \\
\hline TPA & NO & NO & NO & NO & NO & YES \\
\hline
\end{tabular}

Table 1: Congestion control protocols and their support for various Manet scenarios those leads to congestion [6]

\section{CONCLUSION}

The heterogeneity in Manet application scenarios not let to conclude a single and general purpose solution for all possible Manet application scenarios. The solutions discussed in this paper represent the solutions for a subset of the identified problems, which can serve as building blocks for application specific protocol stacks. The categorization of these congestion control models and their Manet application scenarios described in Table1. There is still a lot of scope for the development of new congestion prevention models in Manet protocols. Particularly current experiments can be done to derive a generic cross layer congestion control stack for Manet. The further investigations in this context can consider two remarkable issues:

1. The support hops can be expected to equip with significant memory and computational power in relation to the available network bandwidth

2. And a certain homogeneity of the nodes in different application scenarios

These two considerations offer many opportunities for a protocol design to exploit both cross-layer information and sophisticated support by the intermediate hops. Therefore in this field The experiments can be inevitable that leads to develop a generic cross layer congestion control stacks, which can easily adopt by various manet protocols.

\section{REFERENCES}

[1] D. B. Johnson and D. A. Maltz, Dynamic Source Routing in Ad Hoc Wireless Networks, Mobile Computing, Kluwer Academic Publishers, Vol 353, 1996, pp. 153-181.

[2] Vineet Srivastava, Cross-Layer Design: A Survey and the Road Ahead, IEEE Communications Magazine • December 2005.

[3] Mohammad O. Pervaiz, Michaela Cardei, Jie Wu, Routing Security in Ad Hoc Wireless Networks. Network Security Scott Huang, David MacCallum, and Ding Zhu Du (Eds.) c 2005.

[4] D. Coppersmith and M. Jakobsson, Almost Optimal Hash Sequence Traversal, In Proc. of The Sixth Intl. Conf. on Financial Cryptography (FC 2002), Lecture Notes in Computer Science, Springer 2002.

[5] Z. J. Haas, The Routing Algorithm for the Reconfigurable Wireless Net- works, Proc. of ICUPC 1997, Vol 2, Oct. 1997, pp. 562-566.

[6] ] Christian Lochert, Björn Scheuermann, Martin Mauve, A Survey on Congestion Control for Mobile Ad-Hoc Networks Published at Wiley Wireless Communications and Mobile Computing 7 (5), pp. 655-676, June 2007.

[7] V. Jacobson and M. J. Karels. Congestion Avoidance and Control. Proceedings of SIGCOMM '88, 18(4):314-329, 1988. DOI: http://doi.acm.org/10.1145/205447.205462.

[8] R. Braden. Requirements for Internet Hosts Communication Layers. RFC 1122 (Standard), Oct. 1989. Updated by RFCs 1349, 4379.
[9] M. Mathis, J. Mahdavi, S. Floyd, and A. Romanow. TCP Selective Acknowledgment Options. RFC 2018 (Proposed Standard), Oct. 1996.

[10] Yung Yi and Sanjay Shakkottai. Hop-by-hop Congestion Control over a Wireless Multi-hop Network, 0-7803-83567/04/\$20.00 (C) 2004 IEEE.

[11] Harish Raghavendra, Duc A. Tran. Congestion Adaptive Routing in Ad hoc Networks.

[12] Nishant Gupta, Samir R. Das. Energy-Aware On-Demand Routing for Mobile Ad Hoc Networks, OPNET Technologies, Inc. 7255 Woodmont Avenue Bethesda, MD 20814 U.S.A., Computer Science Department SUNY at Stony Brook Stony Brook, NY 11794-4400 U.S.A.

[13] Laura, Energy Consumption Model for performance analysis of routing protocols in MANET,Journal of mobile networks and application 2000.

[14] LIXin MIAO Jian -song, A new traffic allocation algorithm in $\mathrm{AD}$ hoc networks, "The Journal of China University of Post and Telecommunication”, Volume 13. Issue3. September 2006.

[15] Ahmed S. Nagy, Amr A. El-Kadi, Mikhail N. Mikhail, Published in: Proceeding CNSR '08 Proceedings of the Communication Networks and Services Research Conference IEEE Computer Society Washington, DC, USA (C) 2008

[16] Giovanidis, A. Stanczak, S., Fraunhofer Inst. for Telecommun., Heinrich Hertz Inst., Berlin, Germany This paper appears in: 7th International Symposium on Modeling and Optimization in Mobile, Ad Hoc, and Wireless Networks, 2009. WiOPT 2009

[17] Outay, F.; Vèque, V.; Bouallègue, R.; Inst. of Fundamental Electron., Univ. Paris-Sud 11, Orsay, France This paper appears in: 2010 IEEE 29th International Performance Computing and Communications Conference (IPCCC)

[18] Kumaran, T.S. Sankaranarayanan, V. BSA Crescent Eng. Coll., Chennai, India This paper appears in: 2010 Seventh International Conference On Wireless And Optical Communications Networks (WOCN)

[19] Rashidi, R. Jamali, M.A.J. Salmasi, A. Tati, R. Univ. of Boukan, Boukan, Iran, This paper appears in: AICT 2009. International Conference on Application of Information and Communication Technologies, 2009

[20] Akyol, U.; Andrews, M.; Gupta, P.; Hobby, J.; Saniee, I.; Stolyar, A.; WINLAB, Rutgers Univ., Newark, NJ, This paper appears in: INFOCOM 2008. The 27th IEEE Conference on Computer Communications. 